\title{
¿El pasado importa? Legados institucionales, sociedad civil y burocracia local para la gestión de conflictos socioambientales en Moquegua (2000-2016)*
}

\author{
Aarón Quiñón CÁrdenas**
}

* El autor agradece los comentarios de Omar Coronel a una versión preliminar y las sugerencias realizadas por el revisor o revisora anónima.

** Licenciado en Ciencia Política y Gobierno por la Pontificia Universidad Católica del Perú (PUCP) y miembro del Grupo Interdisciplinario de Enfoque Cualitativos para el Estudio de la Política (GIECEP). Correo electrónico: a.quinonc@pucp.pe

Fecha de recepción: 11/12/18. Fecha de aceptación: 03/04/19 


\title{
¿El pasado importa? Legados institucionales, sociedad civil y burocracia local para la gestión de conflictos socioambientales en Moquegua (2000-2016)
}

\section{RESUMEN}

En el Perú, el reciente boom de commodities y las altas transferencias por canon minero han significado una serie de retos y desafíos para el Estado, en particular, por la gestión de conflictos socioambientales vinculados con las actividades extractivas. No obstante, un balance general muestra fuertes contrastes en el ámbito subnacional, donde, por un lado, encontramos regiones con persistencia de una alta conflictividad social y escasos avances en las mesas de diálogo, mientras que otras regiones evidencian un aprendizaje en la gestión de los conflictos sociales desde la burocracia local y la sociedad civil. ¿Por qué bajo algunos contextos locales la actividad minera ha conducido a serios problemas de conflictividad social y en otros no? La presente investigación centra su atención en el departamento de Moquegua (2002-2016), región dependiente de las rentas mineras que ha mostrado un desempeño positivo en la gestión de conflictos socioambientales y en las mesas de diálogo para la viabilidad de proyectos mineros. Considerando la importancia del contexto regional, el argumento central propone que los legados institucionales y una activa participación de la sociedad civil son factores claves para comprender la trayectoria en la gestión de la conflictividad social. Finalmente, estos factores ayudarán a comprender los retos y desafíos del Estado para la gestión de conflictos en otras regiones.

Palabras clave: recursos naturales, conflictos sociales, sociedad civil, antecedentes institucionales, trayectorias regionales.

\section{Does the past matter? Institutional legacies, civil society and local bureau- cracy for the management of socio-environmental conflicts in Moquegua (2000-2016)}

\begin{abstract}
In Peru, the recent commodity boom and high transfers by mining canon have meant a series of challenges and challenges for the State, in particular, the management of socio-environmental conflicts linked to extractive activities. However, a general balance shows strong contrasts where, on the one hand, we find regions with persistence of social conflicts and little progress in the rounds tables, while other regions show learning in the management of social conflicts from the local bureaucracy and civil society. Why, in some local contexts, mining activity has led to serious problems of social conflict and in others it has not? This research focuses on the department of Moquegua (2002-2016), a region dependent on mining revenues that has shown a positive performance in the management of socioenvironmental conflicts and in the negotiating tables for the viability of mining projects. Considering the importance of the regional context, the central argument proposes that the institutional legacies and active participation of civil society are key factors to understand the trajectory in the management of social conflict. Finally, these factors will help us to understand the challenges of the state for the management of conflicts in other regions.
\end{abstract}

Keywords: natural resources, social conflicts, civil society, institutional legacies, subnational path. 


\section{INTRODUCCIÓN}

El Perú se caracteriza por mostrar un sistema político institucional débil, cuyo sistema económico históricamente ha dependido de las rentas generadas por los recursos naturales, principalmente de las exportaciones mineras. Así, la expansión de la actividad minera en el marco del boom de los commodities durante los últimos quince años ha ocasionado serios impactos en el reciente proceso de descentralización política en el Perú, profundizando una serie de retos y desafíos para el Estado central y un contexto complejo para los recién creados gobiernos regionales (Dargent, Orihuela, Paredes y Ulfe, 2017; Dargent, 2013; Damonte y Glave, 2016). Principalmente, la literatura enfatiza en la explosión de los conflictos socioambientales y la formación de redes de corrupción, así como en la ineficiencia del gasto público (Arellano, 2011a; Arce, 2015; Bebbington, 2012; Maldonado, 2011).

En efecto, durante el período del boom, el incremento de los conflictos sociales se encuentra fuertemente vinculado con las actividades extractivas: más de la mitad de los conflictos son clasificados como socioambientales, y entre 2005 y 2016 la mayoría de los casos fueron ocasionados por la actividad minera. Además, esta situación de conflictividad social evidenció las serias limitaciones y debilidades institucionales del Estado para gestionar este tipo de conflictos en los ámbitos nacional y subnacional, además de claros conflictos de interés al interior del propio Estado (Ormaechea, 2015; Durand, 2016). A raíz de ello, gran parte de la literatura sobre conflictos socioambientales en el Perú ha centrado su atención en casos emblemáticos como Las Bambas (Apurímac), Conga (Cajamarca) y Espinar (Cusco), ilustrando las serias debilidades institucionales y estrategias orientadas desde el Estado para resolución de los conflictos, sea por demandas de contaminación ambiental o por demanda de recursos económicos (Arce, 2015; De Echave, Diez, Revesz, Huber, Tanaka y Lanata, 2009; Paredes, 2016), mientras que estudios cuantitativos identifican variables como pobreza, fragmentación política, distribución de recursos económicos y daños ambientales como factores claves que explican los niveles de conflictos vinculados con la minería (Arce, 2015; Arellano, 2011a).

No obstante, un acercamiento a la evolución en el tiempo de los conflictos permite identificar diferentes trayectorias y contrastes al interior del territorio nacional. En el gráfico 1 se presenta el nivel de conflictividad social en los principales departamentos con actividad minera, donde se aprecia que mientras regiones como Cajamarca, Áncash, Apurímac y Cusco han incrementado notablemente el número de conflictos a lo largo del tiempo, otras, como Arequipa o Moquegua, no escalaron a tales niveles de conflictividad. Incluso la tendencia entre 2007 y 2009 sugería que todas estas regiones alcanzarían una trayectoria similar en la explosión de conflictos asociados a actividades extractivas y varias investigaciones alertaban 
de esta situación a la luz de la «maldición local de los recursos naturales» (ArellanoYanguas, 2011b). Sin embargo, un balance más reciente invita a discutir los retos y desafíos que suponen las rentas y expansión de las industrias extractivas en los gobiernos subnacionales a partir de las trayectorias de regiones con menores niveles de conflictividad en comparación a sus pares.

Gráfico 1. Conflictos sociales en los principales departamentos con actividad minera (2007-2016)

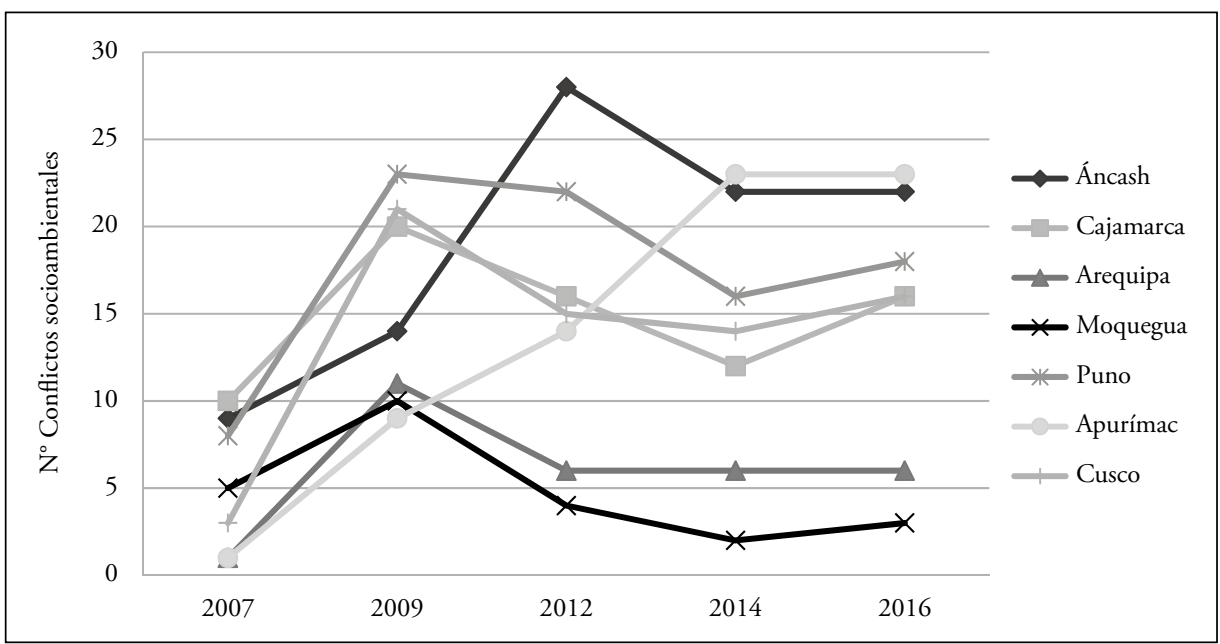

Fuente: Informes mensuales de conflictos sociales - Defensoría del Pueblo (2007-2016).

¿Por qué bajo algunos contextos locales la actividad minera ha conducido a serios problemas de conflictividad social y en otros no? El presente artículo presenta el estudio de caso del departamento de Moquegua, región con una alta dependencia de los ingresos por minería y que, a diferencia de otras regiones, presenta indicadores positivos en desempeño social y económico y es la segunda región con mayor desarrollo humano, con una adecuada provisión de servicios públicos, además de ser considerada la región más competitiva del país. Esta región, además, destaca principalmente por el «éxito» en la implementación de las mesas de diálogo para la viabilidad de proyectos mineros, y ha sido elegida como región modelo para la gestión de conflictos socioambientales.

Considerando la importancia del contexto institucional regional durante el boom de recursos y la expansión de la actividad minera, el presente artículo propone un marco analítico centrado en dos elementos no vinculados en la literatura: legados institucionales y sociedad civil. El argumento central propone que, a partir de los antecedentes institucionales previos al boom de recursos y una participación activa de la sociedad civil, se construyeron factores claves para entender la variación de conflictividad en la trayectoria de los gobiernos regionales. La formación 
de coaliciones y los legados institucionales previos construidos en contextos de baja presión permitirán constreñir trayectorias negativas en contextos de mayor presión, como el boom de recursos y la descentralización.

La metodología empleada para la elaboración del estudio de caso requirió la sistematización y revisión de archivo, principalmente de documentos de gestión municipal y regional elaborados en el período de estudio (2002-2016), así como de informes técnicos presentados por organizaciones locales de cada provincia. Además, se realizó un trabajo de campo para la recolección y triangulación de información primaria mediante veintiún entrevistas semiestructuradas con actores claves de la sociedad civil de dos provincias de la región (Ilo y Mariscal Nieto), incluyendo

a funcionarios públicos de los gobiernos locales y del gobierno regional, así como representantes de organizaciones de la sociedad civil.

El artículo se estructura en cinco secciones, incluyendo esta breve introducción. La siguiente sección discute la literatura sobre conflictos socioambientales en el Perú y presenta el argumento central, proponiendo un marco analítico que resalta la importancia de los legados institucionales y la participación activa de la sociedad civil como elementos centrales para las trayectorias de conflictividad. La tercera sección describe brevemente los antecedentes institucionales en las municipalidades provinciales de Moquegua (1980-2000), analizando la relación entre la sociedad civil y los gobiernos locales antes de la expansión de la actividad minera. La cuarta sección presenta la trayectoria reciente de la región, enfatizando en los legados institucionales en la sociedad civil y la burocracia local y cómo estos marcos condicionan la trayectoria en la gestión de los principales conflictos socioambientales presentados en la región. Finalmente, en la última sección se presentan las conclusiones.

\section{CONFLICTOS SOCIOAMBIENTALES: LEGADOS INSTITUCIONALES Y SOCIEDAD CIVIL}

La conflictividad social en torno a la industria extractiva en el Perú ha sido ampliamente estudiada y la literatura existente presenta suficiente evidencia para mostrar las tensas relaciones entre la actividad minera y las comunidades de su entorno, principalmente por la competencia por recursos naturales (tierras e ingresos económicos), contaminación ambiental e insatisfacción local por una mayor redistribución de rentas (Arce, 2015; Bebbington, 2012).

Por un lado, un grueso de la literatura — principalmente la que emplea métodos cuantitativos - vincula la expansión de los conflictos socioambientales con una versión local de la «maldición de recursos» y el balance general arroja resultados mixtos en el impacto de las industrias extractivas (Arce, 2015; Arellano, 2011a; Orihuela, Huaroto y Paredes, 2014; Orihuela, Pérez y Huaroto, 2018). Por otro lado, numerosos estudios de casos profundizan esta discusión señalando que la relación 
conflictiva no es inmediata ni directa o lineal, y que son recurrentes las demandas por mayores recursos o la defensa del medio ambiente a partir de la contaminación de las empresas mineras, incluyendo el incumplimiento de compromisos asumidos en procesos de negociación (Acuña, 2015; Damonte y Glave, 2016). Por tanto, se presentan diversas motivaciones para la generación de situaciones conflictivas, con objetivos y orígenes distintos para la movilización, y es posible que no en todos los casos exista un rechazo a la actividad minera.

Paredes y De la Puente (2014) señalan que un gran grupo de conflictos socioambientales son de «convivencia». Es decir, las movilizaciones de protesta sociales son mecanismos de presión para visibilizar las demandas y exigencias por autoridades locales y sociedad civil. Por tanto, el objetivo no es la expulsión de la empresa minera de la localidad, sino alcanzar acuerdos para una mejor convivencia entre la actividad minera y las actividades locales, además de alcanzar mejores beneficios económicos. En estos casos, las demandas también se entienden por el incumplimiento de los acuerdos y compromisos realizados por la empresa minera para el apoyo al desarrollo local y mitigación de impactos ambientales.

Las altas transferencias por canon minero y su relación con los niveles de conflictividad social también han llamado la atención de varias investigaciones, dado que en las regiones y localidades con mayores trasferencias de canon se registraba mayor número de conflictos (Arce, 2015; Arellano, 2011a y 2011b). Entre las principales explicaciones por este descontento social se encuentra la reducida capacidad de gasto y gestión de las transferencias de canon minero, cuyos montos exorbitantes no fueron previstos por los gobiernos locales, provocando una insatisfacción de la población local por el incumplimiento de las altas expectativas frente al potencial impacto al desarrollo local con base en el canon (Arce, 2015). En esta línea, Muñoz (2010) identifica que los gobiernos locales con mayores recursos por transferencias no mejoran la provisión de bienes y servicios públicos; al contrario, la estrategia de las municipalidades distritales se traduce en ajustar el gasto de los recursos adicionales e inesperados o no planificados. En otras palabras, hay una mayor ejecución de gasto, pero esto no se refleja en un incremento del bienestar social. Esta problemática se acentúa en los gobiernos subnacionales que perciben transferencias por canon, dado que tienen que gestionar un importante y no planificado incremento de recursos para inversión pública.

De manera general, en gran parte de las investigaciones se encuentra presente la dimensión institucional como un elemento clave para entender el escalamiento de la conflictividad social, prestando atención a la acción contenciosa, a la interacción entre población local y empresa minera o a la debilidad/ausencia de los actores políticos para la gestión del conflicto (Hurtado, 2017). En efecto, el aspecto institucional presenta cambios y continuidades a lo largo de los ciclos mineros, 
fortaleciendo, creando o debilitando instituciones que funcionen como contrapesos a los efectos perversos de la actividad extractiva, evidenciando lo complejidad de la relación entre estos actores y rescatando el valor de los repertorios y marcos institucionales, así como a la red de expertos que funcionan como grupos de soporte (Dargent et al., 2017; Orihuela, 2014). A modo de complejizar la discusión sobre lo «institucional» en el ámbito local, esta puede entenderse desde dos enfoques: (i) como una organización estatal conformada por un cuerpo de funcionarios y burócratas con un conjunto de normas y prácticas internas, y (ii) como marcos de relaciones entre actores locales, incluyendo a funcionarios públicos, autoridades locales y organizaciones de la sociedad civil, entre otros.

Por un lado, es importante destacar el rol de la burocracia local, actores claves para la gestión de las rentas mineras y conflictos sociales, sobre todo en un contexto de escasa planificación estatal para la ejecución de proyectos de inversión, así como en la promoción de la actividad extractiva en territorios con escasa experiencia minera. Ponce y McClintock (2014) identifican que «una mejor capacidad de la burocracia subnacional puede amortiguar los efectos sociales perversos de la maldición de los recursos en el ámbito local» (p. 118). Particularmente, enfatizan en la capacidad de respuesta de la burocracia local hacia las demandas de la sociedad civil, es decir, la capacidad de poder ejecutar las altas transferencias por canon minero en obras de inversion pública y en servicios, principalmente carreteras, hospitales y escuelas. Por tanto, la capacidad de la burocracia local es importante para entender, no solo la explosión de la conflictividad social, sino también la capacidad de gasto de los recursos mineros y el impacto que este podría tener en la provisión de servicios públicos. Incluso, su capacidad y profesionalización durante contextos de presión serían fundamentales para la canalización de demandas locales al construir argumentos «técnicos» con los cuales acompañan y orientan la protesta durante los procesos de negociación.

Por otro lado, distintas investigaciones parten de un enfoque desde el cual «la historia condiciona, pero no determina», donde los legados institucionales sitúan los marcos de acción de los actores públicos y sociedad civil mediante el establecimiento de una serie de repertorios institucionales y procesos de aprendizaje para su relación con la actividad extractiva (Thorp, Battistelli, Guichaoua, Orihuela y Paredes, 2014). En tal sentido, dichos legados operan como «recursos y marcos cognitivos-normativos para las acciones y el activismo de los individuos que integran, reproducen y eventualmente buscan transformar una economía política» (Orihuela, 2013, p. 56). El énfasis en lo institucional parte de la condicionalidad en la trayectoria en la gestión de las rentas extractivas; es importante entender no solo los marcos institucionales, sino la secuencia en el tiempo y la capacidad de agencia de los actores (Battistelli y Guichaoua, 2014; Kurtz, 2013; Orihuela, 2014; Paredes, 2012). 
En particular, un actor central en los conflictos socioambientales son las organizaciones de la sociedad civil y su capacidad de agencia en los procesos de negociación y movilización durante el desarrollo del conflicto, además de su capacidad de demanda para la exigencia del cumplimiento de acuerdos establecidos. Sobre este punto, la literatura reconoce la capacidad de influencia y presión de los actores de la sociedad civil para la canalización y atención de sus demandas, incluso mediante la implementación de políticas públicas y participación directa en los gobiernos locales (Augusto, Dargent y Rousseau, 2017).

En esta línea, McNulty (2013) considera que los nuevos espacios de participación ciudadana creados en el proceso de descentralización han incentivado la inclusión de nuevas organizaciones sociales en los gobiernos locales, principalmente en el presupuesto participativo. Sin embargo, estos actores muestran capacidades y niveles decisionales muy limitados, y en muchos casos son relegados en discusiones más «técnicas» de la gestión local. En el caso de los conflictos sociales mineros, De la Puente (2017) muestra las contradicciones de la inclusión de nuevos actores en las mesas de diálogo, señalando que, a pesar de las movilizaciones y protestas de la sociedad civil organizada y su inclusión en los procesos de diálogo para la canalización de sus demandas, la intervención de las representantes de las organizaciones sociales, en la mayoría de los casos, únicamente alcanzan una "participación cosmética», vale decir, solo se ocupa un asiento en el proceso de negociación sin capacidad de agencia ni participación efectiva. En pocas palabras, la movilización y participación social no bastan para el establecimiento de una trayectoria menos conflictiva. La literatura sugiere que la formación de coaliciones y pactos entre actores locales, principalmente entre actores estatales y sociedad civil, son elementos clave para construir acuerdos políticos y de desarrollo local con cierta continuidad (Augusto, Dargent y Rousseau, 2017), principalmente si los actores de la sociedad civil organizada cuentan con recursos (conocimiento técnico y redes de soporte) y capacidad de movilización social para generar vínculos con los gobiernos locales (Hernández Asensio, 2016).

\section{El argumento}

¿Por qué bajo algunos contextos locales la actividad minera ha conducido a serios problemas de conflictividad social y en otros no? A partir del estudio de caso del departamento de Moquegua (2002-2016), se propone que los antecedentes institucionales previos al boom de recursos y una participación activa de la sociedad civil son factores claves para entender la variación de conflictividad en la trayectoria de los gobiernos regionales en el Perú. El argumento central plantea que la formación de coaliciones locales entre la sociedad civil y los gobiernos locales durante la década de 1980 construyeron una serie de legados institucionales (marcos de relaciones y organizaciones) en un contexto previo al boom de recursos, los cuales permitieron 
constreñir trayectorias negativas y fueron elementos claves para afrontar los retos que genera el boom de recursos y la descentralización política. De manera más específica, se establecieron una serie de repertorios institucionales desde la burocracia local y la sociedad civil que luego se trasladarán a la acción del nuevo gobierno regional para la gestión del conflicto. Así, mediante la presión de las organizaciones de la sociedad civil con experiencia en marcos participativos y con un repertorio de acción contenciosa, se instalaron mesas de diálogo donde el conocimiento de estos actores (sociedad civil y gobiernos locales) permitió constreñir ciertos efectos perversos de la actividad minera.

Figura 1. Legados institucionales y sociedad civil en Moquegua (1980-2018)

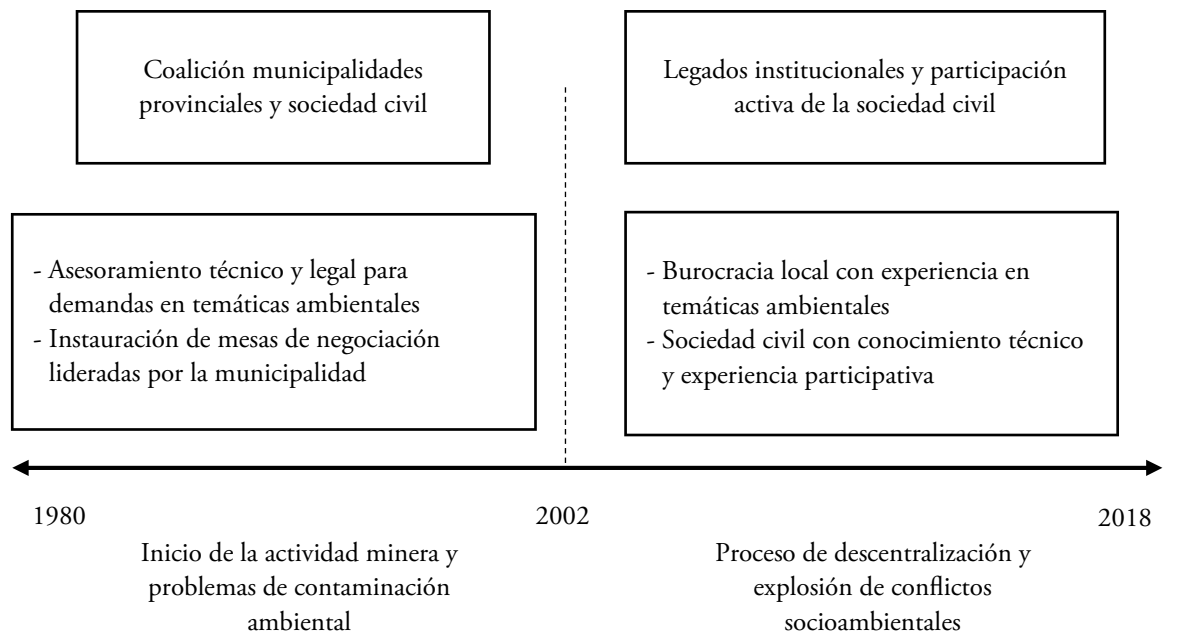

Así, de manera más general, este marco analítico, por un lado, entiende la experiencia como un conjunto de conocimientos (legales y técnicos) de los actores locales (burocracia y sociedad civil), adquiridos a partir de su experiencia en los procesos de negociación y participación previos y asimilados como conocimientos necesarios para su actuación. Por otro lado, la capacidad de presión de la sociedad civil refiere a la posibilidad de incidir en el desarrollo de las actividades de negociación de las organizaciones sociales locales para la inclusión y atención efectiva de sus demandas y reclamos, principalmente mediante acciones colectivas de política contenciosas (movilizaciones y protestas). Esta capacidad de presión es el resultado de una experiencia participativa previa y conocimiento técnico adquirido en su relación con otros actores, como ONG, actores nacionales, entre otros. Finalmente, el traslado de este conocimiento acumulado y actuación colectiva se mantiene en el tiempo debido a la asimilación y continuidad de estos marcos cognitivos y de relaciones como elementos centrales en la dinámica social y política local, vale decir, 
la «rutinización» de la participación en el gobierno local y de planificación estatal de la burocracia local, así como la instauración de estas prácticas en nuevos espacios de poder regional. No obstante, es posible que los legados se debiliten con el paso del tiempo por efectos propios de la «maldición» y procesos políticos externos que acrecienten la presión sobre la actividad extractiva. En este sentido, la agencia de los actores interesados y su interés en mantener esta agenda será crucial para que los legados perduren en el tiempo.

Para concluir el argumento, resulta fundamental destacar la secuencia en el tiempo para la construcción de estos arreglos institucionales: es necesaria su presencia en períodos previos al boom de recursos para poder constreñir y mitigar los efectos perversos durante el período de boom y el proceso de descentralización.

\section{«DE LA PROTESTA A LA PROPUESTA» ${ }^{1}$ : ANTECEDENTES INSTITUCIONALES EN LA GESTIÓN DE CONFLICTOS EN MOQUEGUa (1970-2000)}

En esta sección se presenta una breve reconstrucción de los antecedentes institucionales en las provincias de Ilo y Mariscal Nieto del departamento de Moquegua, atendiendo al nivel de participación de la sociedad civil en los gobiernos locales y en la construcción de un conocimiento compartido de la burocracia local en temáticas ambientales. Como se mostrará, en estos espacios locales se generó una serie de antecedentes y repertorios institucionales, así como una experiencia en protestas y negociaciones lideradas por las municipalidades provinciales y presión de la sociedad civil. Esta experiencia, previa al boom de recursos naturales y el proceso de descentralización, brindará elementos claves para una mejor comprensión de la trayectoria de Moquegua tanto en la gestión de conflictos socioambientales como en la atención de las demandas locales vinculadas con la actividad minera.

\section{Provincia de Ilo}

La provincia de Ilo se encuentra ubicada en la zona costera de la región de Moquegua y es reconocida por registrar una experiencia participativa exitosa en las décadas de 1980 y 1990, caracterizada por una intensa participación de la sociedad civil en los gobiernos locales (Vargas, 1998). En este período, se construyó una coalición local liderada por la municipalidad provincial y organizaciones sociales locales con el objetivo de exigir la remediación de impactos ambientales ocasionados por la fundición de cobre de la Southern Peru Copper Corporation (SPCC), así como la demanda de una planificación para el desarrollo urbano local.

1 Frase atribuida a Julio Díaz Palacios, alcalde provincial de Ilo (1980-1990). 
La fundición de cobre de SPCC inició su instalación en la provincia a inicios de 1950, generando una ola migratoria frente a las expectativas de empleo durante las décadas de 1970 y 1980. A partir del inicio de sus operaciones, SPCC ocasionó serios impactos ambientales en la provincia mediante la emisión de dióxido de azufre, superando largamente los límites de emisión permitidos, y la ciudad fue cubierta por una densa capa de smog que afectó la salud pública de los locales con enfermedades respiratorias y en la piel. Durante este período, la provincia de Ilo era principalmente un campamento minero antes que una ciudad habitable, que incluso incluía la suspensión de actividades protocolares y clases escolares.

Sin embargo, en 1980 ingresa a la municipalidad provincial un grupo político de izquierda liderado por Julio Díaz Palacios, militante de Izquierda Unida (IU) y miembro de la Asociación Civil Labor, una ONG que ingresó en la provincia de Ilo originalmente con un interés político para la formación de un movimiento obrero minero, pero que transitó a una agenda de defensa ambiental y desarrollo urbano. Durante su gestión, Díaz Palacios lideró una serie de movilizaciones frente a la actividad minera y contra la contaminación ambiental ocasionada por SPCC. Estas movilizaciones fueron respaldadas por la sociedad civil organizada, sindicatos y organizaciones vecinales, las cuales contaban con una asesoría técnica en temas ambientales y legales, principalmente de Labor y el CEOP-Ilo. Por ejemplo, entre 1981 y 1987, Díaz Palacios lideró cerca de diez movilizaciones en el ámbito provincial, en coordinación con el Fedip-Ilo, exigiendo la creación de la Comisión Multisectorial de Medio Ambiente, la cual se instaló en agosto de 1987 (Portocarrero y Portugal, 2011)2.

A partir de estas movilizaciones y asesorías técnicas, el gobierno local modificó su estrategia de confrontación a una de negociación mediante la instauración de mesas de diálogo y mesas técnicas, espacios donde la burocracia local aprendió estrategias de negociación y conocimientos en gestión ambiental (Portocarrero, Sanborn, Molina y Loveday, 2007) ${ }^{3}$. El resultado de estas negociaciones no solo fue el establecimiento de acuerdos para la remediación de los impactos ambientales, sino la asunción de compromisos ambientales por SPCC (por ejemplo, incluir monitoreos ambientales participativos y el reporte anual de sus emisiones de dióxido de azufre), los cuales serían supervisados y monitoreados por el gobierno local. Además, la municipalidad inició la elaboración de documentos de gestión ambiental, convirtiéndose

\footnotetext{
2 El hito más importante de protestas se registra el 5 de junio de 1988 en una manifestación pacífica organizada por el Fedip-Ilo y con la participación invitados nacionales e internacionales, incluyendo a delegados del Partido Verde Alemán (Portocarrero y Portugal, 2011).

3 Para mayor detalle de los cambios en la interacción y el proyecto político de Julio Díaz-Palacios, revisar Díaz Palacios (1983).
} 
en la primera provincia en establecer un Plan de Recuperación del Medio Ambiente (PAMA), el cual sería oficializado en 1997.

Díaz Palacios fue reelegido en tres períodos consecutivos durante la década de $1980^{4}$. En 1990, Ernesto Herrera Becerra, quien fuera regidor durante la gestión de Díaz Palacios y también miembro de Labor, fue elegido alcalde provincial (1990-2000) con el objetivo de dar continuidad al modelo participativo iniciado por Díaz Palacios, priorizando las temáticas ambientales y ordenamiento urbano y fortaleciendo los espacios de diálogo (Vargas, 1998). Durante su gestión, Herrera enfrentó un contexto político adverso, en medio de la dictadura de Fujimori, crisis de partidos políticos, y mayores presiones de representación y la articulación social. No obstante, la burocracia local, liderada por el alcalde, enfrentó esta situación mediante la elaboración y aprobación de un plan integral de desarrollo local y la consolidación de una gestión local con base en la participación ciudadana. Estos documentos de gestión fueron elaborados en conjunto con la sociedad civil mediante el modelo participativo y serán las directrices para la ejecución de programas de desarrollo y proyectos de inversión pública, tanto para temáticas ambientales como en desarrollo urbano. Entre estos planes destacan el Plan de Desarrollo Integral de Ilo (PIDI) en 1991, el Plan de Desarrollo Sostenible (PDS) en 1998 y el Plan de Desarrollo Concertado (PDC) en 2000.

A lo largo de las gestiones de Díaz Palacios y Herrera, resultó importante la conformación y participación de las organizaciones sociales de la provincia, quienes impulsaban y demandaban la implementación de estos planes de desarrollo. En particular, destacan el Frente de Defensa de los Intereses de Ilo (Fedip-Ilo), la Comisión de Desarrollo Urbano y el Comité de Manejo Ambiental de la Provincia de Ilo, organizaciones vecinales y diferentes comitivas para resolución de demandas locales. A partir de la participación activa de estas organizaciones, los líderes de la sociedad civil se especializan y adquieren conocimientos técnicos en temáticas ambientales y de desarrollo urbano.

En consecuencia, el resultado de las gestiones de Díaz-Palacios y Herrera fue la elaboración de documentos de gestión y planes de desarrollo, con una burocracia que cuenta con experiencia en temáticas ambientales y de desarrollo urbano y una sociedad civil organizada con una experiencia en un modelo participativo para la canalización de sus demandas, convirtiéndose en un contrapeso importante frente a las empresas extractivas y el gobierno central.

\footnotetext{
4 Continuó su vida política como diputado para el Congreso, espacio donde elevará los reclamos ambientales y de canon minero de Ilo a todo el país, y fue uno de los impulsores de los planes de desarrollo ambiental y el primer Código del Ambiente en 1990.
} 


\section{Provincia de Mariscal Nieto}

La provincia de Mariscal Nieto se ubica en la zona andina de la región y tradicionalmente se ha dedicado a la actividad agrícola y la plantación de viñedos, concentrando su actividad principalmente en los distritos de Moquegua y Torata, dada la presencia de los principales valles agrícolas de la región. Las comunidades campesinas ubicadas en estos distritos iniciaron un proceso de articulación socialcomercial desde 1950 a partir de encuentros comerciales para la venta de productos agrícolas en los sectores más urbanos, incluso en otras regiones. A partir de estas reuniones de campesinos celebradas anualmente en los ámbitos departamental y distrital, estas comunidades empiezan a organizarse y plantear demandas — principalmente económicas - para la mejora en su calidad de vida, impulsadas por el Sistema Nacional de Apoyo a la Movilización Social (Sinamos) y el Fondo de Desarrollo de las Comunidades Campesinas (Fondec) en el marco de la reforma agraria durante la década de $1960^{5}$.

El resultado en el ámbito provincial fue la formación de asociaciones de carácter agrario y campesino organizadas alrededor de la Liga Agraria Provincial José Carlos Mariátegui para el relacionamiento con las entidades del Estado. Estas organizaciones inician una vocación con un alto activismo político y social para la canalización de demandas hacia los gobiernos locales (Cañedo-Argüelles, 2010). Asimismo, dentro de sus objetivos como organización declaran la intención de proporcionar asesoría técnica especializada a sus miembros en temas de salud, educación y recreacional (Portocarrero y Portugal, 2011). Incluso varios líderes campesinos llegaron a ocupar cargos en la municipalidad distrital de Torata durante los años 1970 (CañedoArgüelles, 2010). No obstante, el liderazgo y capacidades técnicas observados en Ilo no se presentan con la misma intensidad en esta provincia.

En 1960 se inicia la actividad minera en el distrito de Torata con la operación de la mina Cuajone por SPCC. Esto originó serios problemas ambientales — principalmente la contaminación de suelos destinada a la actividad agrícola, así como el uso de los ríos como fuente de desechos de minerales- y amenazó directamente el medio de producción agrícola local. Así, mediante protestas y movilizaciones contra SPCC y el gobierno central, se empezó a realizar una serie de litigios contra la empresa minera debido al alto grado de contaminación ambiental por los desechos mineros y relaves en zonas cercanas a los ríos. Al igual que en la provincia de Ilo, las organizaciones campesinas contaron con asesoría legal y programas de capacitación desarrollados por Labor, aunque con menor intensidad y acompañamiento debido a que sus actividades se concentraron en Ilo en ese período.

\footnotetext{
Para mayor detalle sobre la conformación de las comunidades campesinas en esta provincia, revisar Cañedo-Argüelles, Brandis García, Ramírez De Haro y Castaño García (2004).
} 
A partir de este asesoramiento técnico, las organizaciones locales se articularon y contaron con documentos técnicos para poder ejercer sus demandas, principalmente en el Frente de Defensa de los Intereses del Pueblo (Fedip) de Torata - una agrupación que articula a las organizaciones locales del distrito- y las Juntas de Usuarios de Agua de Torata. Estas organizaciones contaron con el soporte y apoyo de organizaciones del distrito de Moquegua, principalmente del Fedip de Moquegua, exigiendo la intervención del Minem para la resolución de esta problemática. No obstante, la articulación con las municipalidades provinciales no fue del todo sólida para la canalización de la protesta y demanda legal, dadas las presiones de SPCC contra los gobiernos locales. Sin embargo, en las oficinas de control de medio ambiente y fiscalización ambiental se realizó un proceso de aprendizaje en la elaboración de documentación para el monitoreo de las actividades de SPCC.

Un hito importante en el proceso de negociación y presión a la empresa minera fue el juicio ambiental realizado contra SPCC ante el Tribunal Internacional de Agua de Holanda entre los años 1988 y 1992, demanda liderada por organizaciones locales y autoridades, así como el acompañamiento de ONG. Así, se alcanzaron algunos acuerdos con la empresa minera para el control de sus residuos y limitar la extracción de aguas subterráneas ${ }^{6}$. Según miembros de la sociedad civil, este acuerdo no fue respetado, lo que ocasionó la pérdida de capacidad productiva del valle de Torata y la contaminación de suelos agrícolas. Esta experiencia marcó definitivamente las relaciones entre la sociedad civil y SPCC.

De esta manera, el resultado del proceso es un continuo aprendizaje en materia jurídica y en diligencias administrativas, tanto en los burócratas como en las organizaciones sociales, durante las acciones realizadas durante veinte años y acompañadas por una asesoría técnica de ONG para los litigios frente a SPCC (Cañedo-Argüelles, Brandis García, Ramírez de Haro y Castaño García, 2004). Por otro lado, las autoridades de las organizaciones locales cuentan con credibilidad por su grado de capacitación y liderazgo, el cual debe estar articulado en programas de desarrollo de alcance local de la municipalidad provincial. Asimismo, varios de los miembros capacitados en este proceso de denuncia contra SPCC alcanzaron gerencias municipales (Cañedo-Argüelles, 2010).

En el ámbito regional, Moquegua logró articular un bloque de protesta frente a los problemas de contaminación ambiental causados por la actividad minera de SPCC, principalmente durante el juicio internacional realizado contra SPCC. Las

\footnotetext{
6 Doris Balvín (1995) reconstruye los argumentos y acuerdos del proceso de demanda contra SPCC, evidenciando las claras afectaciones ambientales y el impacto ocasionado en los ecosistemas por el uso del agua y la emisión de desechos al ambiente. Entre los principales acuerdos destaca un compromiso de inversión de SPCC por 100 millones de dólares para ejecutar el Programa de Recuperación Ambiental.
} 
organizaciones principales son los frentes de defensa y las juntas de usuarios de agua; sin embargo, las que mantienen un mayor nivel de influencia son la Comisión Ambiental Regional y el Frente de Defensa de los Intereses de Moquegua. Un punto clave a destacar es que estas organizaciones centraron su actividad en el distrito de Moquegua, principal ciudad de la región. Estas organizaciones mostraron un gran activismo durante el gobierno autoritario de Alberto Fujimori, período en el que protestaron frente a las prácticas autoritarias del gobierno, aunque estas manifestaciones se fueron debilitando debido a presiones del gobierno central (McNulty, 2011).

Cuadro 1. Legados de las experiencias participativas en el departamento de Moquegua (1970-2000)

\begin{tabular}{llll}
\hline & \multicolumn{1}{c}{ Región } & \multicolumn{1}{c}{ Provincia de Ilo } & \multicolumn{1}{c}{$\begin{array}{c}\text { Provincia de Mariscal } \\
\text { Nieto }\end{array}$} \\
\hline Sociedad civil & $\begin{array}{l}\text { Organización regional } \\
\text { de la sociedad civil } \\
\text { con base en frentes } \\
\text { de defensa, pero no } \\
\text { articulados }\end{array}$ & $\begin{array}{l}\text { Organizaciones } \\
\text { locales con tradición } \\
\text { y experiencia en un } \\
\text { modelo participativo en } \\
\text { la gestión municipal }\end{array}$ & $\begin{array}{l}\text { Gremios campesinos } \\
\text { organizados con } \\
\text { conocimiento técnico y } \\
\text { legal para canalización } \\
\text { de demandas. }\end{array}$ \\
\hline $\begin{array}{l}\text { Gobierno } \\
\text { local }\end{array}$ & $\begin{array}{l}\text { Débil insistencia de } \\
\text { planes de gestión } \\
\text { ambiental sólidos }\end{array}$ & $\begin{array}{l}\text { Documentos de gestión } \\
\text { y planes de desarrollo } \\
\text { elaborados }\end{array}$ & $\begin{array}{l}\text { Programas de desarrollo } \\
\text { y lucha contra la } \\
\text { pobreza, pero no planes } \\
\text { municipales }\end{array}$ \\
\hline $\begin{array}{l}\text { Articulación } \\
\text { entre sociedad } \\
\text { civil y } \\
\text { gobierno local }\end{array}$ & $\begin{array}{l}\text { Débil articulación } \\
\text { entre provincias y } \\
\text { gobiernos locales } \\
\text { para un proyecto de } \\
\text { desarrollo regional }\end{array}$ & $\begin{array}{l}\text { Articulación provincial } \\
\text { densa con base en un } \\
\text { modelo de gestión } \\
\text { participativo }\end{array}$ & $\begin{array}{l}\text { Articulación } \\
\text { provincial media con } \\
\text { la participación de } \\
\text { algunos miembros de } \\
\text { la sociedad civil en la } \\
\text { gestión local }\end{array}$ \\
\hline
\end{tabular}

\section{APLICANDO LO APRENDIDO «BAJO PRESIÓN»: LEGADOS INSTITUCIONALES Y SOCIEDAD CIVIL (2002-2018)}

Los antecedentes institucionales previos y la participación activa de la sociedad civil en Moquegua generaron una serie de condiciones iniciales favorables en un contexto de litigios y negociaciones lideradas por las municipalidades provinciales contra la empresa minera SPCC, aunque con diferentes niveles de asesoramiento y presión de la sociedad civil entre las mismas provincias. No obstante, iniciado el proceso de descentralización y el incremento de la actividad minera en el territorio nacional, las municipalidades provinciales y distritales $-\mathrm{y}$ sobre todo los recientemente creados gobiernos regionales - enfrentaron una serie de problemas y desafíos para el fortalecimiento de sus capacidades en gestión en temáticas ambientales y en la prevención de conflictos sociales asociados a la expansión de la actividad minera. 
Por un lado, sobre la base del modelo económico de exportaciones de materias primas, desde el gobierno central se priorizó la promoción de la inversión extranjera en actividades extractivas y se otorgó un alto número de concesiones para la explotación de recursos mineros. Esta acción estatal de promoción de la actividad minera, emprendida por el Minem y Provinversión, fue acompañada por campañas mediáticas con el fin de ejercer presión sobre los niveles inferiores de gobierno para la gestión de la licencia social que permitiese la aprobación de los instrumentos de gestión ambiental, una de las principales causas del conflicto (Bebbington, 2012).

Por otro lado, las presiones de las empresas mineras representan un serio desafío para las comunidades y gobiernos locales, en particular en el ámbito rural, dada la irresponsabilidad en la gestión de sus residuos mineros, así como en el incumplimiento de los acuerdos de remediación ambiental y social. Así, las localidades afectadas deben negociar las condiciones bajo las cuales convivir con dichas actividades, lo que derivará en una serie de acuerdos que, si bien pueden detener el escalamiento del conflicto, generan otra serie de consecuencias negativas por sus costos sociales y ambientales, además de las presiones para una ejecución de los recursos provenientes del canon minero (Paredes y De la Puente, 2014).

En este marco de mayor presión, a continuación se presentan las dinámicas locales entre la burocracia local y la sociedad civil para la gestión de los conflictos socioambientales y rentas mineras en el marco del proceso de descentralización.

\section{Burocracia local y sociedad civil}

En la provincia de Ilo se ha mostrado cierta continuidad de los modelos de participación ciudadana en la gestión municipal, partiendo de la experiencia de las gestiones previas mencionadas en la sección anterior. En este sentido, la participación ciudadana, mediante las organizaciones de la sociedad civil, continuó siendo el principal medio de presión para la generación de planes de desarrollo de la ciudad y la elaboración del presupuesto público.

Por un lado, las organizaciones sociales de Ilo y las juntas vecinales fueron el principal soporte en la gestión municipal previa de Julio Díaz Palacios y Ernesto Herrera, considerada como la principal referencia que debería ser tomada en cuenta para las actuales gestiones municipales, dado que se ejecutan los proyectos en los cuales estos mismos actores locales participan, presionan y fiscalizan. Además, la experiencia adquirida bajo la participación de los líderes sociales y las capacitaciones de Labor ha servido como fuente de conocimiento para su intervención en la gestión municipal. Incluso, varios de los actuales dirigentes fueron participantes activos de las movilizaciones realizadas en las gestiones de la décadas de 1980-2000 y mantienen estrecha relación con las juntas vecinales y organizaciones locales, espacios 
donde se construyeron y almacenaron estos conocimientos técnicos y legales, mientras que otro grupo de dirigentes surgió a partir del crecimiento de nuevos barrios y juntas vecinales en la provincia, con demandas particulares e intereses de acceso a recursos. De esta manera, si bien el tejido social se mantiene cohesionado, se irá debilitando a medida que aparecen más recursos por canon minero y conflictos en torno a la misma actividad minera. No obstante, este modelo participativo continúa siendo el eje central de la política local, espacio donde los dirigentes con más experiencia tienen como objetivo sostener la estructura social de la provincia.

Por otro lado, la presencia de documentos de gestión y planes de desarrollo previos, que cuentan con el respaldo de la sociedad civil, permitió destinar los recursos provenientes del canon minero a la ejecución de los proyectos de inversión de impacto provincial. De esta manera, se reduciría la probabilidad de destinar el presupuesto a otros proyectos que no fueran identificados como prioridad por los funcionarios de la municipalidad ni por la sociedad civil (McNulty, 2013). En la temática ambiental, la Gerencia de Desarrollo Urbano Ambiental y la Oficina de Ecología y Control Ambiental cuentan con funcionarios públicos con experiencia en la fiscalización y monitoreo ambiental, dado que también participaron durante las gestiones de Díaz Palacios y Herrera y en las mesas técnicas con SPCC. Además, mediante esta presión de la burocracia local se continuó con la elaboración y actualización de los planes de desarrollo local, con Ilo como pionero en la planificación local. Sin embargo, esta planificación no contó con el liderazgo de los nuevos alcaldes, quienes priorizaban la ejecución de obras de corto alcance que les brindaran réditos políticos antes que la planificación institucional, incluso con serias denuncias de corrupción.

Así, la continuidad de la experiencia participativa se evidencia en los procesos de elaboración de los planes de gestión actualizados y, sobre todo, en el Consejo de Coordinación Local (CCL) y en el Presupuesto Participativo (PP) (McNulty, 2011). En estos espacios, la interacción de la sociedad civil y los funcionarios de la municipalidad provincial muestra que la capacidad de articulación previa es importante para el desarrollo de una trayectoria alternativa a la esperada en una provincia con altos recursos mineros.

A lo largo de estos procesos resultó clave la participación de las organizaciones de mujeres, el Fedip-Ilo, la Federación de Pueblos Jóvenes y Asentamientos Humanos (FEDPJAH), los Comités de Gestión Provincial (Transportes, Abastecimiento y Comercialización, Limpieza Pública y Medio Ambiente, Tarifas Municipales) y los Comités de Gestión de Obras. Estas organizaciones fueron miembros activos en los cabildos abiertos y las asambleas populares, en los directorios municipales y en las sesiones del concejo. 
Tabla 2. Principales documentos de gestión en la municipalidad provincial de Ilo (2003-2017)

\begin{tabular}{ll}
\hline Documento de gestión & Año \\
\hline Plan de Desarrollo Concertado (PDC) & 2003 \\
Plan Estratégico Institucional & Actualización trianual \\
Plan Operativo Institucional & Actualización anual \\
Actualización PDC & 2017 \\
\hline
\end{tabular}

Fuente: Portal de acceso a la información pública, municipalidad provincial de Ilo.

En el ámbito regional, la ejecución de proyectos de inversión pública demostró las dificultades de administrar una gran cantidad de recursos en una nueva entidad de gobierno. Antes del inicio del proceso de descentralización, la región Moquegua no había desarrollado una planificación sólida con una mirada regional, dada la concentración de los actores políticos en atender las demandas locales y, sobre todo, concentrarse en las demandas contra SPCC ${ }^{7}$. Además, esta situación se profundizó tras la renuncia de algunos de los funcionarios pertenecientes a la Oficina de Proyectos de Inversión (OPI), un grupo de burócratas que contaba con cierta experiencia de gestión departamental en el Consejo Transitorio de Administración Regional (CTAR) ${ }^{8}$.

No obstante, la necesidad de la planificación para la ejecución de proyectos de alto alcance fue asumida por María Cristala Constantinides, gobernadora regional para el período 2003-2006 y lideresa con una larga trayectoria política en la región, vinculada a Izquierda Unida (IU) y con experiencia de varias gestiones municipales de Moquegua (1996-1998), además de participar en varios momentos en las dinámicas participativas en Ilo durante la década de 1980. Para compensar la salida de los anteriores directivos del CTAR, desde el gobierno regional se conformó un equipo técnico especializado de funcionarios a partir de profesionales locales (principalmente de Ilo), así como de la asistencia de consultorías y ONG que aprendieron rápidamente los procesos administrativos y técnicos para la elaboración de proyectos, en el marco del engorroso procedimiento burocrático del Sistema Nacional de Inversión Pública (SNIP). Estos funcionarios, provenientes de

\footnotetext{
Según los funcionarios públicos entrevistados, la transición con el Consejo Transitorio de Administración Regional de Moquegua (CTAR-Moquegua) fue inadecuada. Un punto débil fue la ausencia de documentos de gestión con planes de desarrollo regional consolidados y aprobados previamente para su implementación.

8 En 1993, durante el gobierno de Fujimori, se crearon los CTAR, organismos públicos descentralizados del Ministerio de la Presidencia para la gestión de las regiones, aunque estas serían muy dependientes del gobierno central.
} 
las municipalidades provinciales, irán rotando entre diferentes gerencias regionales trasladando el conocimiento adquirido durante las gestiones municipales previas, tanto para funciones de planificación como para la implementación de políticas regionales ${ }^{9}$. El resultado al final de la gestión fue una cartera de expedientes de proyectos de desarrollo de cierto alcance regional, principalmente en infraestructura. Así, este período inicial permitió una planificación rápida y la ejecución de algunos proyectos de alcance medio, en especial para el saneamiento básico e infraestructura educativa y de salud.

Además, en los principales espacios para la discusión y repartición del presupuesto regional, tales como el Consejo de Coordinación Regional (CCR), y en especial el Presupuesto Participativo Regional (PPR), participaron activamente autoridades regionales, locales y representantes de la sociedad civil de las tres provincias. Estos espacios son de vital importancia en la región, dado que son los principales mecanismos de relación con la sociedad civil para la elaboración del presupuesto y proyectos. Además, en estos espacios se mostrarán las capacidades técnicas y políticas, tanto de los miembros de la sociedad civil, funcionarios y autoridades en los ámbitos local y regional. Resultó fundamental la experiencia previa de planificación y gestión de los gobiernos locales.

Un punto importante a destacar es que el gobierno regional se encuentra ubicado en la provincia de Mariscal Nieto, en el distrito de Moquegua. Por ello, además de la dinámica descrita anteriormente, el grueso de la atención y de la participación de la sociedad civil será principalmente por organizaciones articuladas en este distrito, en especial de orden barrial, mediante las juntas vecinales. Por ello, las presiones hacia la entidad gubernamental regional se orientarán principalmente al acceso a servicios básicos tales como educación, agua y saneamiento, carreteras, pistas y veredas.

Jaime Rodríguez Villanueva, empresario de Ilo vinculado con la industria pesquera, fue elegido gobernador regional por dos períodos no consecutivos (2007-2010 y 2014-2018). Durante su primera gestión, el presupuesto se incrementó exponencialmente debido a que en 2006 inicia las altas transferencias por canon minero ${ }^{10}$. Ante los réditos políticos que le generaba la ejecución de obras de corto alcance, Rodríguez Villanueva restó prioridad a la elaboración de documentos de gestión como los planes de desarrollo regional. Si bien la gerencia de Planificación y Presupuesto llegó a elaborar estos documentos con la participación

\footnotetext{
9 En el estudio de Arellano Yanguas (2011a), el autor construye un índice de capacidad municipal en regiones mineras, destacando Moquegua por la profesionalización de sus funcionarios y planificación en la gestión.

10 «Las transferencias eran a veces hasta traumáticas. En 2007 nos pasó que nos llegaban hasta 40 millones de soles semanalmente cuando el año anterior solo recibimos 30 millones durante todo el año» (entrevista a gerente del gobierno regional de Moquegua).
} 
de la sociedad civil, no se debió a un liderazgo del entonces gobernador regional. Incluso los propios funcionarios indicaron que los expedientes de los proyectos de alto y medio alcance habían sido elaborados en gran parte por las gestiones anteriores. Por tanto, para Rodríguez la prioridad era la ejecución de las obras antes que la planificación. Si había dinero, era para gastarlo, incluso con serias denuncias de corrupción y con una posición abierta en favor de la minería.

Martín Vizcarra, ingeniero de Ilo y candidato, fue el gobernador regional en el período 2011-2014. Durante la gestión de Vizcarra continuaron las altas transferencias por canon minero y las características de participación ciudadana descritas durante la primera gestión de Villanueva siguieron en la misma línea. Sin embargo, según señalan los funcionarios, con Vizcarra se trató de brindar una mirada más técnica a las obras de infraestructura y de servicios básicos, retomando prioridad la planificación y elaboración de planes de desarrollo con una visión estratégica y territorial. Además, el manejo político de Vizcarra fue importante para conseguir mayores transferencias tanto del gobierno central como de las empresas mineras. Al término de su gestión, considerada una de las más exitosas del país, se consolidaron las intervenciones en políticas educativas regionales, así como la construcción de varios proyectos e infraestructura de salud.

\section{Gestión de conflictos y mesas de diálogo}

A modo de ejemplo se ilustran tres grandes conflictos socioambientales vinculados con la actividad minera en la región, enfatizando los conocimientos en temáticas ambientales y habilidades de negociación adquiridos por la sociedad civil y la burocracia local.

\section{$E l$ «moqueguazo» (2008)}

En junio de 2008 se realizó un paro regional en Moquegua, aproximadamente durante diez días, demandando una mejor redistribución de los porcentajes recibidos por canon minero producidos por las actividades de SPCC y que eran compartidos con la región Tacna. En realidad, esta situación conflictiva se gestó varios ańos antes, dado que, a pesar de producir mayor cantidad de cobre, Tacna percibía el 75\% del canon minero mientras que Moquegua recibía solo el 25\%. Esta situación se explica por el reglamento de canon y regalías promulgado en $2006^{11}$, el cual calculaba el porcentaje de distribución de canon a partir de la cantidad de material de tierra removida en lugar de la proporción de concentrado de extraído, además de aplicar una única cuenta en caso que el titular minero contara con varias concesiones, en este caso SPCC. La aplicación de este reglamento desfavorecía

11 Resolución Ministerial 338-2006-EF. 
a Moquegua, cuya producción y extracción de cobre era mucho mayor sin la necesidad de una mayor remoción de material. Incluso la reducción del canon minero afectaba directamente los presupuestos participativos de regalías desde el año 2006.

En los meses precedentes al estallido del conflicto, el gobierno regional y los gobiernos locales, apoyados por las organizaciones locales, exigieron la instalación de una mesa técnica para la revisión de la normativa que claramente los afectaba, proponiendo la distribución del canon con Tacna en cuentas separadas, y, en especial, cambiar la normativa de canon minero en favor de considerar la proporción de metal extraído por su valor de venta. Sin embargo, desde el gobierno central y el Minem no se obtenía respuesta alguna. No es casualidad que el conflicto por repartición de canon minero surgiera en una región con experiencia en la gestión de este tipo de recursos, los cuales fueron obtenidos por presión de los gobiernos locales de Moquegua en décadas anteriores.

A inicios de junio de 2008, y después de una fallida negociación con la PCM, se declaró un paro indefinido en la región durante dos semanas bajo el liderazgo del Frente de Defensa de los Intereses de Moquegua y los gobiernos locales. Se bloqueó la carretera Panamericana Sur, se aisló la región Tacna de toda forma terrestre de comunicación y se generó escasez de artículos de primera necesidad; además, se paralizaron las actividades escolares. Es importante destacar que la convocatoria a la protesta y movilización partió tanto de la sociedad civil como de las autoridades locales y el acompańamiento de organizaciones locales, lo que hizo posible la actuación de una acción de política contenciosa con el respaldo de los funcionarios especializados en temáticas de gestión minera.

Durante la paralización ocurrieron severos enfrentamientos con la policía, dadas las acciones represivas para el despeje de la carretera. Ante la negativa del gobierno central para la instalación de una mesa de negociación, se recurrió al secuestro de varios efectivos policiales como mecanismo de presión. Esta situación obligó a la PCM a iniciar el diálogo con las autoridades locales, primero para la liberación de los efectivos policiales, y luego, para la discusión de las demandas de repartición de canon. Finalmente, se alcanzó un acuerdo con la firma de un acta de ocho puntos y el levantamiento del bloqueo de la carretera.

El resultado de este proceso fue la modificación en ese mismo año de la Ley de Canon y posteriormente su reglamento ${ }^{12}$, favoreciendo la propuesta presentada por Moquegua para una mejor distribución del canon. Estas modificaciones permitieron que durante el boom de commodities esta región fuese una de las más beneficiadas con las altas transferencias de canon minero y que serán fuente de financiamiento para los proyectos públicos.

12 Ley del Canon 29281 y Decreto Supremo 044-2009 


\section{Mesa de diálogo SPCC}

La mesa de diálogo con la empresa minera Southern ${ }^{13}$ fue iniciada en 2012 bajo la dirección del gobierno regional de Moquegua con la finalidad de atender las demandas de remediación ambiental y social contra SPCC por los daños ambientales ocasionados durante más de cincuenta años de operación en la región. Esta mesa supone un enorme reto para la gestión de conflicto, dados los antecedentes en la relación entre empresa minera, gobiernos locales y sociedad civil, evidenciando un serio resentimiento hacia SPCC por el incumplimiento de varios de los acuerdos establecidos en décadas pasadas y por su negativa de aceptar nuevos compromisos ambientales y sociales. A pesar de estas tensiones previas, se conformaron comisiones de trabajo y se realizaron más de veinte sesiones, en las que se discutieron temáticas como recurso hídrico, medio ambiente y desarrollo sostenible en torno a la actividad minera.

En estas sesiones también participaron organizaciones de la sociedad civil, frentes de defensa, gobiernos locales con asesoría técnica y algunos funcionarios del gobierno central. En estas reuniones se alcanzaron algunos puntos de acuerdo en las negociaciones a pesar de las dilataciones en el proceso ocasionados por SPCC y el gobierno central. No obstante, las negociaciones se suspendieron debido al conflicto en torno a los montos de compensación ambiental, dado que SPCC ofreció un monto de S/ 700 millones a la región de Moquegua, mientras que las autoridades locales consideraron este monto como una ofensa a la región y demandaron que el monto mínimo de compensación fuese mayor de S/ 2080 millones. Al no ceder la empresa minera y no obtener respuesta por el Minem, varias autoridades locales abandonaron la mesa de diálogo y en 2017 se suspendieron las sesiones. Además, miembros de la mesa de diálogo señalaron la falta de liderazgo del gobernador regional Jaime Rodríguez y su poco interés por alcanzar acuerdos o favorecer a la empresa minera.

En la actualidad, la mesa de diálogo con SPCC se encuentra en proceso de reactivación por el electo gobernador regional Zenón Cuevas, quien fuera uno de los principales líderes del «moqueguazo» y expresidente del Fedip-Moquegua. La intención de Cuevas es impulsar las demandas de la sociedad civil y alcanzar un acuerdo con SPCC para los montos de compensación social, incluyendo mayores aportes a obras públicas y mano de obra local.

13 Resolución Ministerial 066-2012-PCM. «Mesa de Diálogo para analizar la problemática hídrica, ambiental, minera y las propuestas de desarrollo del departamento de Moquegua». 


\section{Mesa de diálogo Quellaveco}

El caso más conocido por la gestión del conflicto socioambiental en la región y mediatizada por el gobierno nacional fue la instalación de la mesa de diálogo del proyecto minero Quellaveco, la cual implementada por iniciativa del gobierno regional de Moquegua en 2011. Esta consistió en la instauración de un espacio de diálogo entre la sociedad civil y la empresa minera Anglo American para la obtención de la licencia social del proyecto ${ }^{14}$. Así, mediante la conformación de comisiones en temas ambientales y sociales, en esta mesa participaron representantes de la sociedad civil de Moquegua, autoridades regionales, provincial y distritales, la empresa minera y representantes del Poder Ejecutivo ${ }^{15}$.

Luego de casi catorce meses de discusión, y a partir de las exigencias de la sociedad civil, el resultado fue el establecimiento de una serie de acuerdos para la modificación del EIA del proyecto minero, un mayor compromiso para la contratación de mano de obra local por Anglo American y la creación de un fondo de S/ 1000 millones para la ejecución de medidas de remediación ambiental y programas sociales, con lo que finalmente se alcanzó la gran preciada licencia social. Según los estudios realizados sobre este caso, el «éxito» de esta mesa de diálogo se atribuye al liderazgo del gobierno regional para la atención temprana del conflicto, la asesoría técnica y la capacidad de negociación de la sociedad civil (Hurtado, 2017; PNUD, 2014).

Menos conocida es la trayectoria previa de negociaciones, protestas y movilizaciones del conflicto por Quellaveco originado en 2000 e identificado por la Defensoría del Pueblo en 2007. Once años antes de la instalación de la famosa mesa de diálogo, organizaciones sociales como los frentes de defensa de Moquegua y de Torata exigieron la revisión y modificación del EIA de Quellaveco aprobado por el Minem. Este documento no consideraba las observaciones remitidas por organizaciones comunales y agrarias de Moquegua, que se oponían principalmente al sistema de abastecimiento de aguas subterráneas, con potenciales perjuicios en las escasas fuentes de agua para la agricultura. Edith Arotoma (2013) realiza una valiosa reconstrucción de este proceso, reconociendo la capacidad de manejo de documentos técnicos ambientales de las organizaciones locales, argumentos que resultan válidos para cuestionar la viabilidad ambiental del proyecto minero. De hecho, entre 2000 y 2004, a partir de acciones contenciosas, se presionó para la instalación de una primera mesa de diálogo con participación prioritaria y central de un equipo técnico de PCM, con la finalidad de lograr la derogación del decreto

\footnotetext{
14 Quellaveco es uno de los proyectos mineros de explotación de cobre a tajo abierto. Contempla una inversión aproximada de US\$ 5.300 millones y un proyecto de vida útil de 32 años.

15 Entre los actores destacados se encuentra el presidente del gobierno regional Martín Vizcarra (2010-2014) y Julio Díaz Palacios, cuya participación fue esencial en el manejo de la mesa de diálogo.
} 
que aprobó el EIA. Esta mesa de diálogo técnica fue acompañada por una serie de movilizaciones y huelgas, en tanto no se realizaban las adecuaciones al EIA con respecto al uso de aguas subterráneas. Si bien en 2004 se acordó que la empresa debía revisar su ingeniería hídrica, además de considerar los costos sociales de este proceso, la modificación del EIA presentada en 2008 fue nuevamente observada por la sociedad civil por el uso de las fuentes de agua que alimentan el reservorio de Pasto Grande. El proceso de demandas para las modificaciones del EIA fue similar; no obstante, el resultado del proceso fue la instalación de la conocida mesa de diálogo en 2011, con el establecimiento de acuerdos en materia ambiental e inversión social, además de un mayor protagonismo de las organizaciones de la sociedad civil y lideradas por el gobierno regional.

Por lo tanto, el balance general de la situación conflictiva en esta región evidencia la trayectoria de los conflictos y fortalece el argumento propuesto. Un factor relevante está constituido por el repertorio institucional y la acción contenciosa de protesta social para insistir en la instalación de una mesa de diálogo, la cual fue potenciada y liderada por el gobierno regional y acompañada por la sociedad civil. Ambos actores, burocracia local y sociedad civil, encontraron en los legados institucionales sus marcos de actuación para la canalización de las demandas hasta su institucionalización mediante un decreto o acta, además de mayores exigencias serias hacia la empresa y una pésima gestión del conflicto por el Estado central.

\section{Conclusiones}

La explosión de conflictos socioambientales supone serios retos y desafíos para los gobiernos regionales y locales, actores claves para la canalización y articulación de las demandas locales, sea por demanda de recursos y servicios o denuncias por afectaciones al medio ambiente. El argumento propuesto enfatiza la importancia del contexto regional, la participación activa de la sociedad civil y los legados institucionales que enmarcan el desarrollo de los conflictos mineros y cómo a partir de aprendizajes previos se pueden trazar trayectorias positivas al momento de la negociación y el establecimiento de acuerdos. Así, no solo se resalta el rol de la sociedad civil y las condiciones institucionales, sino que estos factores se construyen desde los antecedentes institucionales y experiencias participativas previas. De tal manera, el contexto y la capacidad de agencia se enmarcan en estos legados institucionales, pero es una condición que se construye desde la experiencia previa y no es tan inmediato. Estos elementos han marcado la trayectoria regional de Moquegua frente a otras regiones, donde la experiencia no es tan positiva y los factores institucionales y sociales no están claramente presentes o no se desarrollaron en esta secuencia de contextos de mayor y menor presión. 
En términos prácticos, también se indica la importancia de la planificación y los legados institucionales construidos en contextos de menor presión, entendiendo la interacción entre una sociedad civil organizada y documentos de gestión elaborados en un marco participativo. Principalmente, los planes de desarrollo locales sintetizan esta concertación entre sociedad civil y Estado, dado que serán los documentos que deberán guiar la ejecución de recursos en momentos de mayor presión.

No obstante, es importante reconocer que los retos y dificultades propios de la administración de los recursos naturales y conflictividad social también han estado presentes en la región Moquegua. Por un lado, al final del período del boom, resulta evidente un desgaste del tejido de la sociedad civil, la priorización de demandas locales y barriales, dependencia de los ingresos por canon minero, casos de corrupción e intentos de redes clientelares, síntomas propios de los desafíos que presenta la abundancia de los recursos. Esta situación permite fortalecer el argumento evidenciando que los retos para la gestión de recursos y conflictos estarán presentes, pero los actores aprenden a comportarse en este marco considerando los legados institucionales. Así, estas condiciones no prescinden de liderazgos y organizaciones fuertes que cuenten con experiencia y que impulsen estos marcos institucionales, destacando autoridades como Díaz Palacios en la experiencia previa local y Martín Vizcarra en la experiencia más reciente, quienes impulsaron estos proyectos políticos con base en marcos institucionales y con gran respaldo de las organizaciones locales.

Finalmente, un rol activo de la sociedad civil será una condición necesaria, pero no suficiente, para constreñir una trayectoria negativa en la gestión de rentas mineras. La comparación con otras regiones evidencia que no solo es necesaria una sociedad civil sólida, sino que esta se encuentre incluida dentro de los marcos institucionales en su relación con el Estado, es decir, importa el tipo de vínculo entre estos dos actores.

En un país caracterizado por serias debilidades institucionales y con una intensa promoción de la actividad minera, la presión de la sociedad civil y la burocracia local, actores contagiados también por los retos que plantea la «maldición», pueden plantear situaciones que generen ciertos contrapesos, pero con la posibilidad de ejercer ciertas limitaciones sobre esta situación conflictiva.

¿Podemos empezar a plantear un path dependence de lo local? La agenda de investigación pendiente invita a pensar la trayectoria y el desarrollo de los conflictos socioambientales en las regiones a partir de la relación entre Estado y la sociedad civil desde las experiencias previas a la descentralización y boom de commodities, y cómo la presencia (o ausencia) de estos legados construyen condiciones institucionales más (o menos) favorables para la gestión de socioambientales. 


\section{REFERENCIAS}

Arce, M. (2015). Movilización por la extracción. En M. Arce, La extracción de recursos naturales y la protesta social en el Perú (pp. 95-212). Lima: Fondo Editorial de la Pontificia Universidad Católica del Perú.

Arellano-Yanguas, J. (2011a). ¿Minería sin fronteras? Conflicto y desarrollo en regiones mineras del Perú. Lima: IEP, PUCP, Universidad Antonio Ruiz de Montoya.

Arellano-Yanguas, J. (2011b). Aggravating the Resource Curse: Decentralisation, Mining and Conflict in Peru. Journal of Development Studies, 47(4), 617-638. https://doi. org/10.1080/00220381003706478

Arotoma Rojas, I.E. (2013). La negociación por el proyecto minero Quellaveco en la región Moquegua (2000-2012). Élites, participación local y conciencia hidrica. Tesis para optar el título de licenciada en Sociología, Pontificia Universidad Católica del Perú, Facultad de Ciencias Sociales, Lima.

Augusto, M.C., E. Dargent y S. Rousseau (2017). Más allá de la capacidad estatal: sociedad civil e implementación de políticas a nivel subnacional en Cusco y San Martín (Perú). Colombia Internacional, 90, 99-125. https://doi.org/10.7440/colombiaint90.2017.04

Balvín, D. (1995). Agua, mineria y contaminación. El caso de Southern Perú. Ilo: Asociación Civil Labor.

Battistelli S. y Y. Guichaoua (2014). ¿Diamantes para el desarrollo? Descifrando la historia de éxito de Botsuana. En R. Thorp, S. Battistelli, J. Orihuela y M. Paredes, Los desafíos de la minería y el petróleo para el desarrollo: lecciones de África y Latinoamérica (pp. 83-132). Lima: Fondo Editorial de la Pontificia Universidad Católica del Perú.

Bebbington, A. (ed.). (2012). Industrias extractivas: conflicto social y dinámicas institucionales en la región andina. Lima: Instituto de Estudios Peruanos.

Cañedo-Argüelles, T. (2010). Del etnodesarrollo al buen vivir: la población aymara se pronuncia. En E. Rey Tristán y P. Calvo González (eds.), XIV Encuentro de Latinoamericanistas Españoles: Congreso Internacional (pp. 2621-2638). Santiago de Compostela: Centro Interdisciplinario de Estudios Americanistas.

Cañedo-Argüelles, T., D. Brandis García, G. Ramírez De Haro y T. Castaño García (2004). Al sur del margen. Avatares y límites de una región postergada. Moquegua (Perú). En T. Cañedo Argüelles (ed.). Madrid: Instituto de Estudios Peruanos. Consejo Superior de Investigaciones Científicas.

Damonte, G. y M. Glave (eds.). (2016). Industrias extractivas y desarrollo rural territorial en los Andes peruanos: los dilemas de la representación politica y la capacidad de gestión para la descentralización. Lima: GRADE.

Dargent, E. (2013). Estado, política y sociedad durante el boom de lo commodities. Argumentos, 5(7). Recuperado de http://revistaargumentos.iep.org.pe/articulos/ estado-politica-y-sociedad-durante-el-boom-de-los-commodities/

Dargent, E., J. Orihuela, M. Paredes y M. Ulfe (eds.) (2017). Resource Booms and Institutional Pathways: The Case of the Extractive Industry in Peru. New York: Palgrave Macmillan. https://doi.org/10.1007/978-3-319-53532-6 
De Echave, J., A. Diez, B. Revesz, L. Huber, M. Tanaka y R. Lanata (2009). Minería y conflicto social. Lima: CIPCA, IEP, CIES, Centro de Estudios Regionales Andinos Bartolomé de Las Casas.

De la Puente, L. (2017). Cuando el diálogo es parte del problema. La institucionalización de las mesas de diálogo en el Perú: el caso de conflicto de Espinar. Lima: Departamento de Ciencias Sociales. Pontificia Universidad Católica del Perú.

Defensoría del Pueblo (2006-2016). Reportes mensuales de conflictos sociales. Lima.

Díaz Palacios, J. (1983). Ilo. La ciudad que quiero ser. Ilo: CIED.

Durand, F. (2016). Cuando el poder extractivo captura el Estado. Lobbies, puertas giratorias y paquetazo ambiental en Perú. Lima: OXFAM.

Hernández Asensio, R. (2016). Los nuevos incas: la economía politica del desarrollo rural andino en Quispicanchi (2000-2010). Lima: IEP. https://doi.org/10.1016/j.exis.2014.11.007

Hurtado, M. (2017). Análisis de la mesa de diálogo entre la sociedad civil de Moquegua, al Estado y la empresa Anglo American Quellaveco 2011-2012: una estrategia de negociación y transformación de conflictos promovida por el gobierno regional de Moquegua 2011. Tesis para optar licenciatura en Ciencia Política y Gobierno, Pontificia Universidad Católica del Perú, Facultad de Ciencias Sociales, Lima.

Kurtz, M.J. (2013). Latin American State Building in Comparative Perspective: Social Foundations of Institutional Order. Cambridge: Cambridge University Press. https://doi. org/10.1017/CBO9781139019668

La República (15 de diciembre de 2012). Espinar negocia con minera Xstrata porcentaje de aporte voluntario y administración. Recuperado de https://larepublica.pe/archivo/ 679976-espinar-negocia-con-minera-xstrata-porcentaje-de-aporte-voluntario-yadministracion

LaRepública(21dejuniode2008).El«moqueguazo»queremecióelsur. Recuperadoel20demayode 2017 de https://larepublica.pe/politica/220608-el-moqueguazo-que-remecio-el-sur

Maldonado, S. (2011). Boom minero y corrupción de funcionarios públicos de los gobiernos locales en el Perú: evidencia de un experimento natural. Berkeley: Centro de Estudios para el Desarrollo y la Participación, Universidad de California.

McNulty, S. (2011). Voice and Vote: Descentralization and Participation in Post-Fujimori. California: Standford University Press.

McNulty, S. (2013). Participatory Democracy? Exploring Peru's Efforts to Engage Civil Society in Local Governance. Latin American Politics \& Society, 55(3), 69-92. https://doi.org/10.1111/j.1548-2456.2013.00203.x

Merino, R. (2015). The politics of extractive governance: Indigenous peoples and socioenvironmental conflicts. Extractive Industries and Society, 2(1).

Muńoz, P. (2010). ¿La política importa? Los determinantes políticos de eficiencia del gasto municipal. Lima: Consorcio de Investigación Económica y Social.

Orihuela, J. (2013). Instituciones y cambio institucional: repensando la maldición de los recursos desde los nuevos institucionalismos y la experiencia peruana. Politai, 4(6), 47-62. 
Orihuela, J.C. (2014). Construcción y reingeniería de «la buena gobernanza» en Chile. En R. Thorp, S. Battistelli, Y. Guichaoua, J. Orihuela y M. Paredes, Los desafios de la minería y el petróleo para el desarrollo. Lecciones de África y Latinoamérica (pp. 45-82). Lima: Fondo Editorial de la Pontificia Universidad Católica del Perú.

Orihuela, J.C., C. Huaroto y M. Paredes (2014). Escapando de la maldición de los recursos locales: conflictos socioambientales y salidas institucionales. Lima: CIES.

Orihuela, J.C., C.A. Pérez Cavero y C. Huaroto (2018). Does fiscal windfalls increase mining conflicts? Not always. Extractive Industries and Society (July). https://doi. org/10.1016/j.exis.2018.07.010

Ormaechea, I. (2015). Aportes para una politica de transformación de conflictos con equidad desde las regiones. Lima: Consorcio de Investigación Económica y Social.

Paredes, M. (2012). Shaping state capacity: a comparative historical analysis of mining dependence in the Andes, 1840s-1920s. Tesis doctoral, Universidad de Oxford, Departamento de Desarrollo Internacional.

Paredes, M. (2016). The glocalization of mining conflict: Cases from Peru. Extractive Industries and Society, 3(4), 1046-1057. https://doi.org/10.1016/j.exis.2016.08.007

Paredes, M. y L. De la Puente (2014). Protestas y negociaciones socioambientales. El caso de las industrias extractivas. En G. Damonte y G. Vila (eds.), Agenda de investigación en temas socioambientales (pp. 75-105). Lima: Cisepa-PUCP.

PNUD (2014). Experiencia de diálogo, transformación de conflicto y construcción de consensos. Sistematización de la Mesa de Diálogo de Moquegua. Lima.

Ponce, A. y C. McClintock (2014). The explosive combination of inefficient local bureaucracies and mining production: evidence from localized societal protests in Peru. Latin American Politics and Society, 56(3), 118-140. https://doi.org/10.1111/j.15482456.2014.00243.x

Portocarrero, F. y A. Portugal (2011). Historia de un compromiso con el desarrollo sostenible. Tres décadas de vida institucional de la Asociación Civil Labor (1981-2011). Lima: Asociación Civil Labor.

Portocarrero, F., C. Sanborn, O. Molina y J. Loveday (2007). De la protesta a la propuesta: la Asociación Civil Labor. En F. Portocarrero, C. Sanborn y L. Camacho, Moviendo montañas: empresas, comunidades y ONG en las industrias extractivas (pp. 121-193). Lima: Centro de Investigación de la Universidad del Pacífico.

Thorp, R., S. Battistelli, S., Y. Guichaoua, J. Orihuela y M. Paredes (2014). Los desafíos de la minería y el petróleo para el desarrollo. Lecciones de África y Latinoamerica. Lima: Fondo Editorial de la Pontifica Universidad Católica del Perú.

Vargas, C. (1998). Desarrollo local y participación politica en Ilo. Nuevas formas de articulación y representación social y politica. Lima: IEP. 